\title{
Berufliche Rehabilitation bei erworbener Hirnschädigung Patienteninformation
}

Herr Schmitz hatte einen Schlaganfall. Auch nach fast 2 Jahren geht er zur Logopädie und Krankengymnastik. Sein rechter Arm bleibt gelähmt. Häufig fallen ihm die Wörter nicht mehr ein. Manchmal verwechselt er sie, ohne dass er es bemerkt. Früher musste er beruflich viel telefonieren. Herr Schmitz fragt sich, ob er jemals wieder arbeiten kann...

Lena hatte einen Fahrradunfall auf dem Weg zur Uni. Neben diversen Knochenbrüchen erleidet sie ein Schädel-HirnTrauma. Körperlich geht es ihr bald besser. Dennoch ist nichts mehr wie vorher. Das Erlernen neuer Dinge fällt ihr schwer. Ihre Lehrveranstaltungen $\mathrm{zu}$ planen und $\mathrm{zu}$ koordinieren bereitet ihr große Probleme. Sie wollte immer studieren, einen Plan B gibt es nicht...

Die Folgen einer erworbenen Hirnschädigung betreffen besonders die Bewegungsfähigkeit (Motorik), die Kognition (z.B. Gedächtnis, Konzentration, Planung) oder die Sprache (Aphasie) bzw. das Sprechen (Dysarthrophonie, Sprechapraxie).

Nicht selten stellt sich die Frage, ob der alte Arbeitsplatz erhalten werden kann oder ob eine (Wieder-) Aufnahme einer beruflichen Tätigkeit möglich ist.

\section{Leistungen zur Teilhabe am Arbeitsleben \\ $\nabla$}

Für die Finanzierung der beruflichen Rehabilitation ist u.U. ein anderer Kostenträger zuständig als für medizinisch-therapeutische Behandlungen. Die gesetzlich verankerten Leistungen zur Teilhabe am Arbeitsleben (LTA) umfassen die wegen einer Behinderung erforderlichen Hilfen zur Erhaltung, Besserung und Wiederherstellung der Erwerbsfähigkeit.

\section{Kostenträger der beruflichen Rehabilitation (LTA, SGB IX § 6 )}

1. Träger der (Kriegs-) Opferversorgung/-fürsorge

2. Träger der gesetzlichen Unfallversicherung

3. Träger der öffentlichen Jugendhilfe

4. Träger der gesetzlichen Rentenversicherung

5. Bundesagentur für Arbeit

6. Träger der Sozialhilfe

\section{Berufliche Förderung bei Verlet- zungen des Gehirns $\nabla$}

Zu Beginn einer Maßnahme der beruflichen Rehabilitation erfasst der Leistungserbringer die individuellen Fähigkeiten, Erfahrungen und Interessen. Für Menschen mit erworbenen Hirnschädigungen haben sich ambulante, wohnortnahe Maßnahmen bewährt. Das wichtige soziale Umfeld bleibt dadurch erhalten. Ein Vorteil ist außerdem, dass die berufliche Reha eng mit der medizinischen verzahnt werden kann. Der regelmäßige Austausch mit den behandelnden Therapeuten (z. B. Logopäden, Ergotherapeuten, Neuropsychologen) und dem Anbieter der beruflichen Reha ist unbedingt sinnvoll. Dadurch können Übungserfolge unmittelbar auf die berufliche Tätigkeit bezogen umgesetzt oder Vorfertigkeiten trainiert werden.

Die Vorgehensweise in der ambulanten beruflichen Rehabilitation orientiert sich immer am Einzelnen und ist nicht einheitlich festgelegt. Beispiele sind:

- psychologische und/oder berufspädagogische Eignungstestung

- berufsvorbereitendes Training, z.B. Organisation der Arbeitsabläufe

- begleitete Arbeitserprobung in einem Betrieb vor Ort oder beim (ehemaligen) Arbeitgeber

- „Training on the job“, d.h. in einem Betrieb wird gemeinsam mit einem Jobcoach über einen längeren Zeitraum ein Nischenarbeitsplatz entwickelt und erprobt. Dieser wird ausgehend von den individuellen Fähigkeiten und Neigungen geschaffen (s.g. Unterstützte Beschäftigung, § 38a SGB IX)

- Aus- oder Weiterbildung, individuell begleitet und unterstützt

- Beratung des Arbeitgebers im Hinblick auf die Gestaltung des Arbeitsplatzes und bezogen auf zusätzliche finanzielle Leistungen für Menschen mit einer (Schwer-) Behinderung

- gemeinsame Auswertung der praktischen Erprobung, Bewertung der momentanen Fähigkeiten des Rehabilitanden bezogen auf eine berufliche Tätigkeit

- ggf. Nachbetreuung zur weiteren Stabilisierung am betrieblichen Arbeitsplatz

\section{Antragsverfahren}

$\nabla$

Zunächst wird ein Antrag bei einem Kostenträger auf Leistungen zur Teilhabe am Arbeitsleben gestellt. Dieser leitet den Antrag ggf. an den zuständigen Träger weiter. Nach Feststellung des Reha-Bedarfs überlegt der zuständige Reha-Berater mit dem Rehabilitanden, welche Leistungen zur beruflichen Teilhabe für ihn infrage kommen. Alternativ kann statt Sachleistungen Geld in Form eines Persönlichen Budgets zur Verfügung gestellt werden. Dieses wird nach Zielvereinbarung mit dem Kostenträger für die Wunschleistung eingesetzt. Unterstützung für das Antragsverfahren bieten Gemeinsame Servicestellen, deren Kontaktdaten im Internet abrufbar sind.

\section{Daniela Päßler-van Rey, Aachen}

überreicht durch Thieme und 